\title{
The possibility of the calibration/validation of the GOCE data with the balloon-borne gradiometer
}

\author{
J. B. Zieliński ${ }^{1}$ and M. S. Petrovskaya ${ }^{2}$ \\ ${ }^{1}$ Space Research Centre, Polish Academy of Sciences, Warsaw, Poland \\ ${ }^{2}$ Central Astronomical Observatory, Russian Academy of Sciences, Pulkovo, Russia
}

\begin{abstract}
A new calibration/validation experiment for GOCE mission is proposed. Simultaneously with the satellite mission another gradiometer will be flown on the board of the stratospheric balloon on the altitude $20-40 \mathrm{~km}$. The measurements can be compared with satellite data. The advantage of this method is that the same functionals are compared - gravity gradients. The post-mission external calibration/validation is possible more directly than through the comparison with the ground truth gravity anomalies or geoid undulation. The calibrating gradiometer is less sensitive, but thanks to the altitude difference, compatible in precision with the orbiting GOCE gradiometer. Analytical procedure of the downward continuation is presented which permits comparison of these observables, supported by numerical examples. The threshold for the precision of the calibrating gradiometer is indicated. Similar comparison can be done between GOCE and GRACE missions.
\end{abstract}

\section{Introduction}

The calibration and validation of results of space missions devoted to the gravity field investigation is of great importance because the measuring instrumentation is working on limits of its capability. In the case of the GOCE mission a number of approaches have been suggested, and among them are the use of ground based gravity data and the data from other gravity missions (Koop at al., 2001). It is understood that the GOCE measurements can be compared with the external data set converted into gravity gradients. Fortunately during the current years we have a sequence of missions which can be used for comparison, e.g. CHAMP and GRACE. The optimal approach would be to perform measurements with an other independent gradiometer in the same points as GOCE gradiometer. This is hardly possible, but we can consider the application of the airborne gradiometer flying at the altitude of $40-30 \mathrm{~km}$ on the board of a stratospheric balloon. In this study we discuss possibilities of the comparison between the missions by the upward continuation procedure. We concentrate on theoretical aspects of the

Correspondence to: J. B. Zieliński (jbz@ @bk.waw.pl) experiment, leaving aside for the time being more technical questions like measurement error characteristics.

\section{Balloon-borne Gradiometer (BBG) and GRACE Mis- sion}

In the Institute for Space Physics CERN (Italy) the economic and flexible accelerometer ISA (Italian Space Accelerometer) has been developed which can be applied in a range of missions. Its sensitivity is better than $10^{-12} \mathrm{~g} / \sqrt{ } \mathrm{Hz}$. The next application of it will be the ESA Beppi Colombo mission aimed to Mercury (Iafolla et al., 2000; Iafolla and Nozzoli, 2002). But another possibility of application is the construction of the gravity gradiometer. It will consist of a cluster of accelerometers connected to perpendicular axes yielding the gravity gradient tensor components. An appropriate geometrical arrangement of accelerometers and baselines allows to perform the measurement of all elements of the gravity gradient tensor, in principle. The expected precision of the measurement is of the level of $0.01 \mathrm{EU}$ (1 Eötvös Unit $=10^{-9} \mathrm{~m} \mathrm{~s}^{-2} / \mathrm{m}$ ). The mission, which was proposed for this system, was the free fall drop from the stratospheric balloon. Such implementation was proposed by the Italian Space Agency ASI as a project called G-zero or GIZERO (Iafolla et al., 1997). Essential for this project is a payload that allows to achieve zero gravity with residual noises $10^{-13} \mathrm{~g} / \sqrt{ } \mathrm{Hz}$ for about 25-30 s. These circumstances became an inspiration for the study by (Zieliński and Lorezini, 1998), with the main task to consider the possibility of the application of the Frascati accelerometr system inside the GIZERO capsule to the Earth gravity field measurement. This project is called Free Falling Gradiometer. Unfortunately, at the time being, the GIZERO project is suspended. However, another possibility can be considered, to fly the gradiometer on the stratospheric balloon drifting with the wind along the trajectory at altitude ca. $40 \mathrm{~km}$ (Balloon borne Gradiometer - BBG). Such flights are realized from the Italian Balloon Launch Base Milo, in Sicily. With the wind the balloon can fly westward from Sicily, along the African coast, reaching eventually the Atlantic Ocean. The trajectory could have about $2000 \mathrm{~km}$, and the flight duration about $24 \mathrm{~h}$. 
For this kind of measurement the ISA gradiometer could be used after some modifications enabling the housing of the instrument in the balloon gondola and the filtering of accelerations resulting from the motion of the balloon. However, this option has not yet been tested experimentally and requires further development.

A completely different possibility of the validation of GOCE offers the mission GRACE. It was launched on 17 March 2002, its successful performance so far permits to expect the positive outcome of the whole mission when it terminates after five years. It is composed of two sub-satellites separated by ca. $200 \mathrm{~km}$, following each other along the same circular orbit with an altitude of about $500 \mathrm{~km}$. The data from this mission can provide the "comparison from above" for GOCE. In both cases the procedure of the upward continuation has to be applied. Let us consider the analytical and the numerical approach, the second one based on collocation using as the signal the components of the gravity gradient of the field described by a model:

$T(r, \theta, \lambda)=\frac{\mu}{a} \sum_{n=2}^{N} \sum_{m=-n}^{n}\left(\frac{a}{r}\right)^{n+1} \bar{C}_{n, m} \bar{Y}_{n, m}(\theta, \lambda)$.

In this expression $T$ denotes the disturbing potential, where:

$\bar{Y}_{n, m}(\theta, \lambda)=\bar{P}_{n,|m|}(\cos \theta) Q_{m}(\lambda)$

$Q_{m}(\lambda)=\left\{\begin{array}{cc}\cos m \lambda, & m \geq 0, \\ \sin |m| \lambda, & m<0,\end{array}\right.$

$\bar{P}_{n,|m|}(\cos \theta)$ and $\bar{C}_{n, m}$ are the fully normalized associated Legendre functions and $r$ is the geocentric distance, $\theta$ and $\lambda$ are the polar angle and the longitude, a the semimajor axis of the normal ellipsoid, and the mass of the Earth multiplied by the gravitational constant.

\section{Analytical solution}

In the GOCE satellite gradiometry mission the diagonal second order derivatives of the disturbing potential $T_{x x}, T_{y y}$, and $T_{z z}$, coud be inferred. It is expected that from them a new geopotential model will be constructed of high accuracy and resolution. This procedure can be performed e.g. by the space-wise approach if there are analytical relations between the spectral coefficients of the observables and the geopotential coefficients $\bar{C}_{n, m}$. Such relations were derived for by the authors in Petrovskaya et al. (2001).

The observables in the balloon mission can be presented in terms of the geopotential coefficients. If $\bar{C}_{n, m}$ evaluated from GOCE measurements are substituted into the analytical expressions for the balloon observables then the discrepancies can be estimated between the real balloon data and the same quantities generated by the GOCE geopotential model. This procedure is developed in the present paper.

A gradiometer inside a balloon consists of two accelerometers measuring the differences between the components of the accelerations of two proof masses keeping a constant distance between each other (the baseline of a gradiometer).
The differences between the acceleration components provide the differences between the components of the gravity disturbances at the locations of two masses. We assume here that the non-gravitational effects can be eliminated with sufficient accuracy.

By dividing such a difference by the distance between the masses, the corresponding second order potential derivative is estimated in the centre of the baseline. This procedure provides the balloon gradiometry data.

It is convenient to use the local ellipsoidal reference frame $\bar{x}, \bar{y}, \bar{z}$ with the origin in the centre of the gradiometer, where $\bar{z}$ is extended outward along the ellipsoidal normal, $x$ is directed to the north and $\bar{y}$ points to the east, completing the left-handed system. There are the following relations between the partial derivatives of the Earth's disturbing potential $T$ with respect to coordinates $\bar{x}, \bar{y}, \bar{z}$ and the spherical coordinates $r, \theta, \lambda$ (Heck, 1990):

$T_{\bar{z}}=T_{r}-\frac{1}{r} e^{2} \sin \theta T_{\theta}$,
$T_{\bar{x}}=-T_{\theta}-e^{2} \sin \theta \cos \theta T_{r}$,
$T_{\bar{y}}=\frac{1}{r \sin \theta} T_{\lambda}$.

Here $e$ is the first eccentricity of the normal ellipsoid.

In the right hand sides of Eq. (2) we confine ourselves by taking into account only the principal terms and omit the terms proportional to $e^{2}$. They will be considered in the future, if necessary. Formula (1) has the same form if the normalised coefficients and spherical functions are changed to the non-normalised ones. After this transformation, we substitute the series Eq. (1) in the right hand sides of Eqs. (2). It gives

$T_{\bar{z}}=-\frac{\mu}{a^{2}} \sum_{n=2}^{N} \sum_{m=-n}^{n}(n+1)\left(\frac{a}{r}\right)^{n+2} C_{n, m} P_{n,|m|}(\cos \theta) Q_{m}(\lambda)$,

$T_{\bar{x}}=-\frac{\mu}{a^{2}} \sum_{n=2}^{N} \sum_{m=-n}^{n}\left(\frac{a}{r}\right)^{n+2} C_{n, m} \frac{d P_{n,|m|}(\cos \theta)}{d \theta} Q_{m}(\lambda)$,

$T_{\bar{y}}=-\frac{\mu}{a^{2}} \sum_{n=2}^{N} \sum_{|m|=1}^{n} m\left(\frac{a}{r}\right)^{n+2} C_{n,-m} \frac{P_{n,|m|}(\cos \theta)}{\sin \theta} Q_{m}(\lambda)$,

Equations (4) and (5) contain either the singularity $\sin ^{-1} \theta$ for $\theta=0, \pi$ or the derivative of the Legendre function. Suitable transformations will be performed in order to exclude these expressions. In Petrovskaya (1996) a number of known transformation formulas are given. We shall apply formulas (45), (47), (48) and (50):

$\frac{d P_{n, 0}}{d \theta}=-P_{n, 1}$

and for $1 \leq|m| \leq n$

$\frac{d P_{n,|m|}}{d \theta}=-\frac{1}{2} P_{n,|m|+1}+\frac{1}{2}(n+|m|)(n-|m|+1) P_{n,|m|-1},(6)$ 
$\frac{d P_{n,|m|}}{d \theta}=|m| \cot \theta P_{n,|m|}-P_{n,|m|+1}$,

$\frac{d P_{n,|m|}}{d \theta}=n \cot \theta P_{n,|m|}-\frac{1}{\sin \theta}(n+|m|) P_{n-1,|m|}$.

From the last two equations it is derived

$m \frac{P_{n,|m|}}{\sin \theta}=\frac{n-|m|+1}{n+|m|+1} \frac{d P_{n+1,|m|}}{d \theta}+\frac{n+1}{n+|m|+1} P_{n+1,|m|+1}$.

Then from Eqs. (6)-(8) and (9) follows

$$
\begin{aligned}
& |m| \frac{P_{n,|m|}}{\sin \theta}=\frac{1}{2} P_{n+1,|m|+1} \\
& \quad+\frac{1}{2}(n-|m|+1)(n-|m|+2) P_{n+1,|m|-1}, m \neq 0
\end{aligned}
$$

From Eqs. (4)-(8) and (10) it is obtained, after introducing normalized quantities,

$$
\begin{aligned}
T_{\bar{z}} & =-\sum_{n=2}^{N} \sum_{m=-n}^{n} \bar{C}_{n, m} t_{\bar{z}}^{n, m}, \\
T_{\bar{x}} & =\sum_{n=2}^{N} \sum_{m=-n}^{n} \bar{C}_{n, m} t_{\bar{x}}^{n, m}, \\
T_{\bar{y}} & =\sum_{n=2}^{N} \sum_{m=-n}^{n} \operatorname{sign} m \bar{C}_{-n, m} t_{\bar{y}}^{n, m} .
\end{aligned}
$$

In the last equation $m \neq 0$ and $\operatorname{sign} m=m /|m|$. In Eq. (11), the functions on the right hand side are given by:

$$
\begin{gathered}
t_{\bar{z}}^{n, m}=\frac{\mu}{a^{2}}(n+1)\left(\frac{a}{r}\right)^{n+2} \bar{P}_{n,|m|}(\cos \theta) Q_{m}(\lambda), \\
t_{\bar{x}}^{n, 0}=\frac{\mu}{\sqrt{2} a^{2}} a_{\bar{x}}(n, 0) \bar{P}_{n, 1}, \\
t_{\bar{x}}^{n, m}=\frac{\mu}{2 a^{2}}\left(\frac{a}{r}\right)^{n+2}\left[a_{\bar{x}}(n,|m|) \bar{P}_{n,|m|+1}(\cos \theta)\right. \\
\left.\quad-b_{\bar{x}}(n,|m|) \bar{P}_{n,|m|-1}(\cos \theta)\right] Q_{m}(\lambda), \\
t_{\bar{y}}^{n, m}=\frac{\mu}{2 a^{2}}\left(\frac{a}{r}\right)^{n+2}\left[a_{\bar{y}}(n,|m|) \bar{P}_{n+1,|m|+1}(\cos \theta)\right. \\
\left.\quad+b_{\bar{y}}(n,|m|) \bar{P}_{n+1,|m|-1}(\cos \theta)\right] Q_{m}(\lambda) .
\end{gathered}
$$

In two last expressions $m \neq 0$, and the coefficients are:

$$
\begin{aligned}
& a_{\bar{x}}(n,|m|)=\sqrt{(n+|m|)(n+|m|+1)}, \\
& b_{\bar{x}}(n,|m|)=\sqrt{1+\delta_{|m|, 1}} a_{\bar{x}}(n,-|m|), \\
& a_{\bar{y}}(n,|m|)=\sqrt{\frac{2 n+1}{2 n+3} \sqrt{(n-|m|+1)(n+|m|+2)},} \\
& b_{\bar{y}}(n,|m|)=\sqrt{1+\delta_{|m|, 1}} a_{\bar{y}}(n,-|m|) .
\end{aligned}
$$

Here $\delta_{|m|, 1}=1$ for $|m|=1$ and $\delta_{|m|, 1}=1$ for $|m| \neq 1$. After calculating the quantities (12)-(13) at the locations of the two proof masses they are substituted at the right hand sides of Eq. (11), as well as the coefficients $\bar{C}_{n, m}$ of a geopotential model. By performing the summation of series, the differences between the components of the gravity disturbances at the ends of the baseline can be calculated and then from them the corresponding values of the diagonal derivatives will be evaluated.
By comparing the values of the derivatives which are measured by the balloon gradiometer and the same derivatives which are estimated with the use of formulae (11)-(13) from the geopotential model, derived from the GOCE mission, the validation of the satellite gradiometer can be studied on the basis of the balloon data.

The problem of such a comparison can be strictly solved by applying the results from (Petrovskaya et al., 2001), where simple basic relations were derived between the geopotential coefficients $\bar{C}_{n, m}$, entering (11) and the spherical harmonic coefficients of GOCE observables. Of these observables, the most important are the diagonal derivatives $T_{x x}, T_{y y}, T_{z z}$, which refer to the local north-oriented triad $\{x, y, z\}$ centred at the satellite, where $z$ is directed radially outward, $x$ points to the north and $y$ to the west (the right handed system). There are linear relations between the derivatives $T_{x x}, T_{y y}, T_{z z}$ and the derivatives $T_{u u}, T_{v v}, T_{u v}$, referred to the local orbital frame in which GOCE measurements will be performed. These relations were derived independently at least by two authors (Vermeer, 1990; Belikov, 1994).

Let us assume that for each potential derivative from GOCE mission a set of observational data is created, regularly gridded over a mean orbital sphere of radius $r=r_{s}$. For simplicity, we shall write simply $r$ instead of $r_{s}$. Then the corresponding spectral coefficients of the potential derivatives can be evaluated, either by the least squares adjustment or by the numerical quadrature technique. Then the above mentioned basic relations allow to recover the geopotential coefficients $\bar{C}_{n, m}$ from GOCE data.

The procedure of application of the basic relations is as follows.

The potential derivatives are presented in form of truncated spherical harmonic series:

$$
\begin{aligned}
& T_{z z}=\sum_{n=2}^{N} \sum_{m=-n}^{n} \bar{R}_{n, m} \bar{Y}_{n, m}(\theta, \lambda), \\
& T_{x x}=\sum_{n=0}^{N} \sum_{m=-n}^{n} \bar{H}_{n, m} \bar{Y}_{n, m}(\theta, \lambda), \\
& T_{y y}=\sum_{n=0}^{N} \sum_{m=-n}^{n} \bar{G}_{n, m} \bar{Y}_{n, m}(\theta, \lambda) .
\end{aligned}
$$

where the coefficients and spherical functions are fully normalised.For the coefficients of $T_{z z}$ there is the relation

$\frac{\mu}{a^{3}}(n+1)(n+2)\left(\frac{a}{r}\right)^{n+3} \bar{C}_{n, m}=\bar{R}_{n, m}$

from which a geopotential model can be easily constructed. For the other two diagonal derivatives the following basic relations were derived in (Petrovskaya et al., 2001):

$$
\begin{gathered}
\bar{a}_{n, m} \bar{C}_{n-2, m}-\bar{b}_{n, m} \bar{C}_{n, m}+c_{n, m} \bar{C}_{n+2, m} \\
=a_{n, m} \bar{H}_{n-2, m}-b_{n, m} \bar{H}_{n, m}+c_{n, m} \bar{H}_{n+2, m} \\
-\bar{d}_{n, m} \bar{C}_{n-2, m}+\bar{c}_{n, m} \bar{C}_{n, m} \\
=a_{n, m} \bar{G}_{n-2, m}-b_{n, m} \bar{G}_{n, m}+c_{n, m} \bar{G}_{n+2, m} \\
|m|=0,1 \ldots, N, \quad n=|m|,|m|+1, \ldots, N .
\end{gathered}
$$


Here $\bar{a}_{n, m}, \bar{b}_{n, m}$, etc., are numerical constants similar to Eq. (13), which depend only on $n$ and $m$. Each of the above relations represents a set of linear equations with respect to the potential coefficients $\bar{C}_{n, m}$ for a fixed $m$ and variable $n$. The set of Eqs. (17) can be solved by a recurrent procedure with respect to $\bar{C}_{n, m}$ in terms of the coefficients $\bar{G}_{n, m}$ of GOCE mission observable $T_{y y}$. The set Eq. (16), containing three unknown potential coefficients, can be presented in form of a linear matrix equation. The latter can be solved with respect to $\bar{C}_{n, m}$ in terms of the coefficients $\bar{H}_{n, m}$ of observable $T_{x x}$.

The geopotential coefficients $\bar{C}_{n, m}$ recovered from each of Eqs. (15)-(17) will be substituted in the right hand sides of Eqs. (11). In this way the derivatives $T_{\bar{x}}, T_{\bar{y}}$ and $T_{\bar{z}}$ will be estimated from GOCE observables at the positions of two probe masses in the balloon gradiometer. It will allow to perform the calibration/validation of the satellite gradiometer.

\section{Least Squares Collocation solution}

Collocation enables local solutions including application of discrete measurements. The problem that must be solved here is the comparison of measurements done in two different places in space, at the altitudes differing by at least $200 \mathrm{~km}$. Similar problem has been discussed by Albertella et al. (2000). To solve this problem we can use the approach proposed by Arabelos (2001), Arabelos and Tcherning (1998) modified according to the needs of the applied technique.

Let us remind after (Zieliński, 1974) the basic formulae of the prediction method by collocation. We assume that an "observation" $x$ consists of the "signal" $s$ and the "noise" $n$. In vector notation we get

$\boldsymbol{x}=\boldsymbol{s}+\boldsymbol{n}$.

Then, we can calculate the signal in the point $P$ :

$s_{P}=\mathbf{C}_{\mathbf{P}} \mathbf{C}^{-\mathbf{1}} \boldsymbol{x}$

where: $\quad \mathbf{C}_{\mathbf{P}}$ - covariance matrix between the signal in $P$ and observations;

$\mathbf{C}$ - covariance matrix between observations; $\boldsymbol{x}$ - vector of observations.

A similar expression allows to estimate the prediction error:

$\varepsilon_{P}=C_{0}-\mathbf{C}_{\mathbf{P}} \mathbf{C}^{-1} \mathbf{C}_{\mathbf{P}}^{\mathbf{T}}$

where: $\quad C_{0}$ - variance of the signal.

According to the classical formulation the covariance function of the disturbing potential $T$ is:

$$
\begin{aligned}
& K_{n}(\Psi)=\Sigma_{n=2} k_{n} P_{n}(\cos \Psi)\left(\frac{R^{2}}{r_{p} r_{q}}\right)^{n+1}, \\
& k_{n}=\frac{G M}{R} \Sigma_{m=0}\left(\bar{C}_{n m}^{2}+\bar{S}_{n m}^{2}\right),
\end{aligned}
$$

where $\bar{C}_{n m}^{2}, \bar{S}_{n m}^{2}$ are fully normalized coefficients of the disturbing potential.

From Eq. (21) expressions for covariance functions between any functionals can be derived. For example, Latka (1978) presents the following formula for the covariance function between two vertical components of the gravity gradient:

$$
\begin{aligned}
& \cos \left(T_{P}^{z z}, T_{Q}^{z z}\right)=\left(\frac{1}{r_{P} r_{Q}}\right)^{2} \\
& \quad \sum_{n=0}^{N} k_{n}(n+1)^{2}(n+2)^{2}\left(\frac{R^{2}}{r_{P} r_{Q}}\right)^{n+1} P_{n}(\cos \phi) .
\end{aligned}
$$

In the same work we can find the expressions for all the combinations of the components of the gravity gradient.

Because the repetition of measurements in the same place by two independent methods is clearly impossible, let us try to compare the measurements made at two different levels: the orbit level and the balloon level. This comparison can be done by two steps: first, the upward continuation procedure enabling to transfer the values of a gradient from the low to the orbital altitude along the radial direction, second, to calculate the gradient from the satellite measurements at the same point. Suppose we have a number of satellite measurements at points $S_{1}, S_{2}, \ldots S_{q}$. Also, we have balloon measurements at points $B_{1}, B_{2}, \ldots B_{p}$. Each balloon measurement is projected by the upward continuation on the sphere of the satellite orbit and we get points $S B_{1}, S B_{2}, \ldots S B_{p}$. For this points we calculate gradient values from satellite measurements using expression (19) and compare with the projected balloon values. In this way the satellite measurements could be verified.

Just now we are trying to go test this procedure by using the set of the simulated data generated by Ilk et al. (2000). This is a file containing the global coverage of the 30 days of observations. In our collocation approach we have to work with a the local or regional solution, therefore it was necessary to extract a sub-set limited in space.

\section{Accuracy requirements}

In the upward continuation process the most significant effect is related to the attenuation of the amplitudes of terms of the geopotential model with increasing height. For the second gradient it is proportional to Sneeuw (2000):

$$
\left(\frac{R}{r}\right)^{n+3} \text {. }
$$

If we denote the geocentric distance to the satellite as $r_{s}$ and the geocentric distance to the balloon as $r_{b}$, we can write:

$$
\left(\frac{R}{r_{b}}\right)^{n+3} /\left(\frac{R}{r_{s}}\right)^{n+3}=\left(\frac{r_{s}}{r_{b}}\right)^{n+3}=\alpha .
$$

Taking $h_{b}=40 \mathrm{~km}$ and $h_{s}=250 \mathrm{~km}$ we get the function $\alpha$ growing quickly with $n$. For $n=70$ we get $\alpha \sim 10$, while for 
$n=140 \alpha$ attains 100 . For $n=360$ the parameter $\alpha$ exceeds $10^{5}$. It means that the gradiometers having the same sensitivity could sense different frequencies at the altitude of the balloon and on the satellite orbit. On the contrary, in order to observe the terms of the same frequency we need different accuracy of the two instruments. If we want to measure and to compare terms of the order close to $n=70$, then we must take $\alpha=10$, what means that the balloon instrument can be 10 times less sensitive than the satellite gradiometer. For the terms of the order $n>140$, the balloon measurements could be even $10^{2}$ less sensitive.

\section{Concluding remarks}

In the situation of the GOCE mission, we will get very reliable information covering the lower part of the spectrum of the geopotential derived from the CHAMP and GRACE data. As concerning GRACE, the optimal resolution in the interval $50<n<120$ is expected (Jekeli, 2000). Therefore, the most interesting part for the calibration by balloon is for $n>100$. From Eq. (23) we can calculate that for $n=100, \alpha \approx 30$. Supposing the GOCE's gradiometer sensitivity equal to $5 \times 10^{-3} \mathrm{EU} / \sqrt{ } \mathrm{Hz}$ we may assume that the airborne measurements at the balloon altitude with the accuracy not worse than $1.5 \times 10^{-1} \mathrm{EU}$ can provide very interesting material for comparison and validation. This is encouraging for the further work on the balloon gradiometer system development.

Theoretical considerations presented above might be applied to the comparison of other measurements done at two different levels. If we take the GRACE data, they are located on the quasi-sphere $250 \mathrm{~km}$ higher than GOCE. Then, the high sensitivity of the GOCE gradiometer is compatible with the sensitivity of the satellite-to-satellite tracking system of GRACE

Acknowledgement. This work has been supported by the grant from the Polish Committee for Scientific Research, No. 8 T12E 05121.

\section{References}

Albertella, A., Miligaccio, F., and Sanso, F.: Application of the concept of biorthogonal series to a simulation of a gradiometric mission, IAG Symposia, Vol. 114, edited by Sanso, F., Geodetic Theory Today, Springer Verl., 1995.

Albertella, A., Miligaccio, F., and Sanso, F.: Direct and local comparison between different satellite missions for the gravity field on-the-fly, IAG Symposia, Vol. 121, edited by Schwarz, K. P., Geodesy Beyond 2000 - The Challenges of the First Decade, Springer Verl., 2000.

Arabelos, D.: Calibration of satellite gradiometer data by ground gravity data, Presented at IAG 2001 Scientific Assembly - Vistas for Geodesy in the New Millenium, Budapest, Hungary, 2001.

Arabelos, D. and Tcherning, C. C.: Calibration of Satellite Gradiometer Data Aided by Ground Gravity Data, J. Geodesy, 72, 617-625, 1998

Belikov, M.: Global gravity field modelling from space observables: complete simulation / recovery experiment, Delft Univ. of Technology, Final Project Rep., Delft, 1994.
Heck, B.: On the linearized boundary value problems of physical geodesy, Dept. Geod. Sci. and Surv., Ohio State University, Columbus, Ohio, 1990.

Iafolla, V., Lorenzini, E., Miliukov, V., and Nozolli, S.: GIZERO: New Facility for Gravitational Experiments in Free Fall Gravitation and Cosmology, 3, 1-10, 1997.

Iafolla, V., Mandiello, A., and Nozzoli, S.: The High Sensitivity Italian Spring Accelerometer (ISA) for Fundamental Physics in Space, Adv. Space Res., 25, 6, 1241-1244, 2000.

Iafolla, V. and Nozzoli, S.: Italian Spring Accelerometer (ISA) a high sensitive accelerometer for "Bepi Colombo" ESA CORNERSTONE, Planet. Space Sci., in press, 2002.

Ilk, K. H., Kusche, J., and Visser, P.: Simulation Study: CHAMP, GRACE, GOCE. IAG-Section II, CD-ROM., 2000.

Jekeli, Ch.: Calibration/validation methods for GRACE, IAG Symposia, Vol. 121, edited by Schwarz, Geodesy Beyond 2000 - The Challenges of the First Decade, Springer Verl., 2000.

Koop, R. and Bouman, J.: Review of Calibration Methods for GOCE Data, Presented at IAG 2001 Scientific Assembly - "Vistas for Geodesy in the New Millennium" Budapest, Hungary, 2-7 September, 2001.

Latka, J.: The Use of Satellite Gradiometry for Determination of Gravity Anomaly, Beitraege zur Astronomischen Geodaesie und Geodynamik. Inst. Fuer Physikalische Geodaesie der Technischen Hochschule Darmstadt, 1978.

Lemoine, F. G., Kenyon, S. C., Factor, J. K., Trimmer, R. G., Pavlis, N. K., Chinn, D. S., Cox, C. M., Klosko, S. M., Luthcke, S B., Torrence, M. H., Wang, Y. M., Williamson, R. G., Pavlis, E. C., Rapp, R. H., and Olson, T. R.: Geopotential model EGM96. NASA/TP-1998-206861, Goddard Space Flight Center, Greenbelt, 1998 .

Lorenzini, E. C., Fuligni, F., Zieliński, J. B., Cosmo, M. L., Grossi, M. D., Iafolla, V., and Rothman, T.: Baloon-Released Gravitation Experiments in Free Fall, Adv. Space Res., 14, 2, 113-118, 1994.

Mueller, J.: Die Satellitengradiometriemission GOCE, Deutsche Geodaetische Kommission, Reihe C. Nr. 541, 2001.

Petrovskaya, M. S.: Optimal Approach to the Investigations of the Earth's Gravitational Field by Means of Satellite Gradiometry, Artificial Satellites, 31, 1, 1-23, 1996.

Petrovskaya, M. S., Vershkov, A. N., Volfson, G. B., and Zieliński, J. B.: Simulation of the spectra of GOCE observables from a geopotential model, Presented at IAG 2001 Scientific Assembly - "Vistas for Geodesy in the New Millennium" Budapest, Hungary, 2-7 September, 2001.

Petrovskaya, M. S., Vershkov, A. N., and Zieliński, J. B.: Recovering the Earth's potential spectral characteristics from GOCE mission, Adv. Space Res., 30, 2, 221-226, 2002.

Schrama, E. J. O.: Collinear Track Calibration of the GOCE Gravity Gradiometer, Presented at IAG 2001 Scientific Assembly - "Vistas for Geodesy in the New Millennium” Budapest, Hungary, 2-7 September, 2001.

Sneeuw, N.: A semi-analytical approach to gravity analysis from satellite observations, Deutsche Geodaetische Kommission, Reihe C. Nr. 527, 2000.

Vermeer, M.: Observables quantities in satellite gradiometry, Bull. Geod., 64, 347-361, 1990.

Zieliński, J. B.: Vertical Sounding of the Earth's Gravity Field, Deutsche Geodaetische Kommission, Reihe A, 81, 1974.

Zieliński, J. B. and Lorenzini, E.: Study of the Free Falling Gravimeter Experiment, Final Report to Polish Academy of Sciences and Smithsonian Institution. Warsaw, 1998. 